\title{
Mechanism of spreading of the highly related neurofibromatosis type 1 (NF1) pseudogenes on chromosomes 2, 14 and 22
}

\author{
Mirjam Luijten ${ }^{1}$, YingPing Wang ${ }^{2}$, Blaine T Smith ${ }^{2}$, Andries Westerveld ${ }^{1}$, Luc J Smink ${ }^{3}$, \\ Ian Dunham ${ }^{3}$, Bruce A Roe ${ }^{2}$ and Theo JM Hulsebos ${ }^{1}$

\begin{abstract}
${ }^{1}$ D epartment of Human Genetics, Academic Medical Center, University of Amsterdam, The Netherlands; ${ }^{2}$ Department of Chemistry and Biochemistry, University of Oklahoma, Norman, OK, USA; ${ }^{3}$ Sanger Centre, Wellcome Trust Genome Campus, Hinxton Hall, Cambridge, UK
\end{abstract}

Neurofibromatosis type 1 (NF1) is a frequent hereditary disorder that involves tissues derived from the embryonic neural crest. Besides the functional gene on chromosome arm 17q, NF1-related sequences (pseudogenes) are present on a number of chromosomes including $2,12,14,15,18,21$, and 22 . We elucidated the complete nucleotide sequence of the NF1 pseudogene on chromosome 22. Only the middle part of the functional gene but not exons21-27a, encoding the functionally important GAP-related domain of the NF1 protein, is presented in this pseudogene. In addition to the two known NF1 pseudogenes on chromosome 14 we identified two novel variants. A phylogenetic tree was constructed, from which we concluded that the NF1 pseudogenes on chromosomes 2, 14, and 22 are closely related to each other. Clones containing one of these pseudogenes cross-hybridised with the other pseudogenes in this subset, but did not reveal any in situ hybridisation with the functional NF1 gene or with NF1 pseudogenes on other chromosomes. This suggests that their hybridisation specificity is mainly determined by homologous sequences flanking the pseudogenes. Strong support for this concept was obtained by sequence analysis of the flanking regions, which revealed more than $95 \%$ homology. We hypothesise that during evolution this subset of NF1 pseudogenes initially arose by duplication and transposition of the middle part of the functional NF1 gene to chromosome2. Subsequently, a much larger fragment, including flanking sequences, was duplicated and gave rise to the current NF1 pseudogene copies on chromosomes 14 and 22. European Journal of Human Genetics (2000) 8, 209-214.

Keywords: neurofibromatosis type 1; pseudogene; sequence analysis; evolution

\section{Introduction}

Neurofibromatosis type 1 (NF1) is a frequent autosomal disorder with an incidence of approximately 1 in 3000 births. The disease involves tissues derived from the embryonic neural crest and the most important clinical features of the disorder are multiple neurofibromas, café-au-lait spots and Lisch nodules of the iris. The NF1 gene, located on chromoso-

Correspondence: Dr TJM Hulsebos, Department of Human Genetics, Academic Medical Center, University of Amsterdam, Meibergdreef 15, 1105 AZ Amsterdam, The Netherlands. Tel: +31 20 5665170; Fax: +31206918626; E-mail: t.j.hulsebos@amc.uva.nl

Received 27 August 1999; revised 18 November 1999; accepted 22 November 1999 me17q11.2, consists of 59 exons distributed over $350 \mathrm{~kb}$ of genomic DNA and encodes a protein of 2818 amino acids, called neurofibromin. ${ }^{1}$ Neurofibromin contains a central domain showing homology to the catalytic domain of mammalian GTPase-activating proteins (GAPs). ${ }^{2}$ The GAPrelated domain (GRD), encoded by exons21-27a, stimulates the intrinsic GTPase-activity of the proto-oncogene ras p21. By converting ras $\mathrm{p} 21$ from the GTP- to the GDP-bound form, the activity of ras p21 is down-regulated.

In recent years, numerous NF1-related sequences (pseudogenes) have been identified on various chromosomes, including $2,12,14,15,18,21$, and $22 .^{3-11}$ Most of the NF1 pseudogenes have been mapped to pericentromeric regions. 
The chromosome2 NF1 pseudogene has been localised in region $2 q 21$, which is known to contain the remnant of an ancestral centromere. ${ }^{12}$ NF1-related sequences are classified as non-processed pseudogenes, as partial sequence analysis revealed a conserved exon-intron organisation. On chromosomes 14 and 15, respectively two and three different NF1 pseudogene copies have been found..$^{3-5,7,10,11}$

In this study, we investigated the mechanism of spreading of NF1 pseudogenes, in particular those on chromosomes2, 14, and 22. In a previous report, ${ }^{9}$ we showed that cosmids containing the chromosome22 NF1 pseudogene only crosshybridise with the NF1 pseudogene regions on chromosomes 2 and 14 . No hybridisation was detected with NF1-related loci on other chromosomes or with the functional NF1 gene. To explain this apparent lack of crosshybridisation, we suggested that the NF1-related Ioci on chromosomes 2, 14, and 22 form a subset of NF1 pseudogenes that are highly related to each other. To demonstrate this, we determined exactly which exons are represented in the NF1 pseudogenes on chromosomes 2, 14, and 22 and we established their evolutionary relationship. In addition, we sequenced regions flanking the NF1 pseudogenes on these chromosomes and show that these are highly homologous to each other.

\section{Materials and methods Southern blot analysis}

Exons of the functional NF1 gene were amplified by PCR using as template DNA extracted from a human $\times$ mouse hybrid cell line with chromosome17 as the sole human contribution (GM10498B, NIGMS Human Genetic Mutant Cell Repository, Camden, NJ, USA). PCR primers and conditions were according to Li et al. ${ }^{1} \mathrm{PCR}$ products were recovered from low melting temperature agarose, labelled with $\alpha-\left[{ }^{32} \mathrm{P}\right] \mathrm{dCTP}$ and hybridised to Southern blots containing digests with HindlII of total human DNA, and of DNA extracted from the chromosome17-only hybrid cell line, from a chromosome22-only human $\times$ mouse hybrid cell line (PgMe25NU), from a chromosome14-only human $\times$ mouse cell line (Wegroth B3), from a chromosome2-only human $\times$ hamster cell line (GM10826B, NIGMS Human Genetic Mutant Cell Repository, Camden, NJ, USA), from a mouse-only cell line (Wehi-TG) and from a hamster-only cell line (A3). Signal densities were quantified by laser scanning densitometry (Phosphorimager, Molecular Dynamics, Sunnyvale, CA, USA) using Imagequant Software v.30. Cell lines PgMe25NU, Wegroth B3 and Wehi-TG originated from and were kindly provided by A Geurts van Kessel, University of Nijmegen, Nijmegen, The Netherlands.

\section{Screening of libraries}

A chromosome 2 cosmid library, LL02NC02 (Lawrence Livermore National Laboratory, Human Genome Center, Livermore, CA, USA; UK HGMP Resource Centre, Hinxton, Cambridge, UK), was screened for NF1-related sequences using PCR products of exons 15, 17, and 19a of the functional NF1 gene as probes as described previously. ${ }^{13}$ A Human Female PAC Library, RPCI6 no. 709 (Pieter de Jong, Roswell

Functional NFl gene

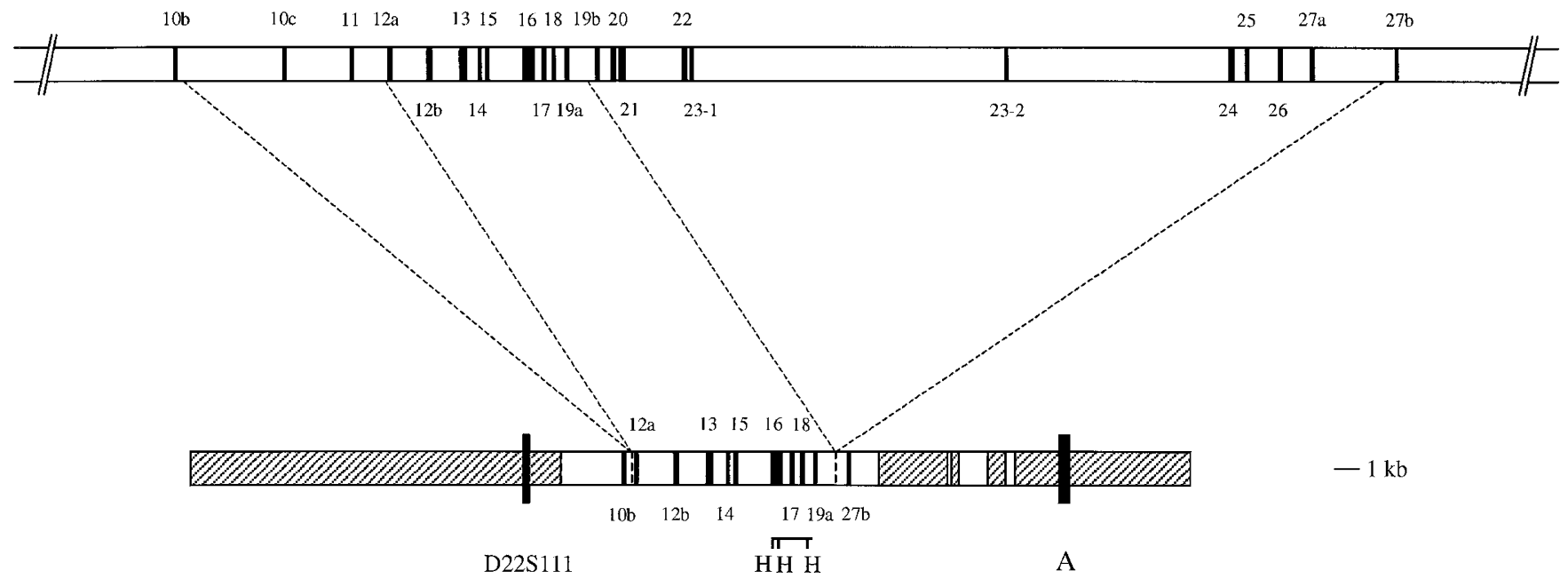

\#22 NF1 pseudogene

D22S111

Figure 1 Genomic organization of the NF1 pseudogene on chromosome22 in cosmid 29A9 (GenBank Accession No. AC005374) and the corresponding part of the functional NF1 gene. Exons and introns are denoted by filled and open boxes, respectively. Hatched boxes indicate sequences in cosmid 29A9 that are not related to those in the functional NF1 gene. Cosmid $29 A 9$ has an insert of $41958 \mathrm{bp}$. The NF1-homologous region starts at position 15686 and ends, except for a few additional regions of homology to intron 27b, at position 28934. The positions of region D22S111 (from 14122 to 14381) and of region A (from 36421 to 36848) are indicated. H: HindIII restriction sites in exon 16 and intron 18 (see text for details). 
Park Cancer Institute; Resource Center of the German Human Genome Project at the Max-Planck-Institut for Molecular Genetics), was screened in an analogous way.

\section{PCR amplification and DNA sequencing}

Primer sequences for amplification of region A (see Figure 1) were as follows: forward, 5'-CTGTTCTTCAGTTAGAGCTTATTACTC-3' and reverse, 5'-CTCACATACATTATCTCATTCATACT-3'. Both this region and marker D22S111 were amplified using standard PCR conditions.

Sequencing of cosmids 29A9, 29D 1 and $127 \mathrm{~F}^{9}$ was performed according to procedures described previously. ${ }^{14}$ The derived sequences were compared to the sequence of the NF1 locus ${ }^{15}$ and the cDNA sequence according to the NNFF International NF1 Genetics Analysis Consortium (http:/ /www.nf.org/nflgene/nflgene.cDNAtext.html). Selected regions in and flanking the NF1 pseudogenes on chromosomes 2 and 14 were sequenced on an Applied Biosystems Model 377 automated sequencer using the Big Dye Terminator Cycle Sequencing Ready Reaction Kit (Perkin Elmer, Foster City, CA, USA)

\section{Phylogenetic analysis}

Phylogenetic analyses were performed on the exon-related sequences of the NF1 pseudogenes on chromosomes2, 14 and 22 and the corresponding sequences of the functional NF1 gene using the PHYLIP package (http://evolution.genetics.washington.edu/phylip.html). The maximum parsimony tree was obtained by branch-and-bound algorithm and contiguous gaps were recoded as a single character prior to analysis. For the neighbour-joining method, the statistical support for each branch was determined in terms of branch length and bootstrap analysis, using 1000 iterations.

\section{Results}

In a previous study, we isolated cosmids that contain NF1-related sequences on chromosome 22. ${ }^{9}$ FISH analysis with these cosmids using high stringency conditions not only showed a hybridisation signal in the pericentromeric region of chromosome arm $22 \mathrm{q}$, but also in the pericentromeric region of chromosome arm $14 q$ and in region $2 q 21$, the latter known to contain the remnant of an ancestral centromere. As these cosmids did not hybridise to other NF1-related loci or to the functional gene on chromosome arm 17q, we suggested that the NF1-related loci on chromosomes 22,14 , and 2 are related to each other and form a subset of NF1 pseudogenes.

To determine which NF1-related exons are present in the pseudogene on chromosome22, we hybridised PCR products of a large number of exons of the functional NF1 gene to Southern blots with digests of DNA extracted from a chromosome22-only human $\times$ mouse cell line. Humanspecific hybridising fragments were only detected for exons10b, 12a-19a, and 27b (data not shown). The same results were obtained for cosmids29A9, 29D1, and 127F6, suggesting that the entire chromosome $22 \mathrm{NF1}$ pseudogene is contained within these cosmids.

Next we determined the complete nucleotide sequence of these cosmids (GenBank Accession Nos AC002471, AC003064, AC005374). Only the middle part of the functional NF1 gene is represented in this pseudogene (Figure 1). Exons10c-11 and 19b-27a and their intervening sequences are absent. As sequences of introns19a and 27a of the functional NF1 gene are known, ${ }^{1,15}$ the transition between their homologous sequences in the pseudogene could be determined exactly. At the 5 ' end of the chromosome 22 NF1 pseudogene, intron 10a is represented by its last $2594 \mathrm{bp}$. At the $3^{\prime}$ end, the first $2934 \mathrm{bp}$ of intron $27 \mathrm{~b}$ are present. However, the representation of intron $27 \mathrm{~b}$ is disrupted three times by sequences that are not related to those in the functional NF1 gene, consisting of $2875 \mathrm{bp}, 299 \mathrm{bp}$ and 694 bp, respectively (Figure 1).
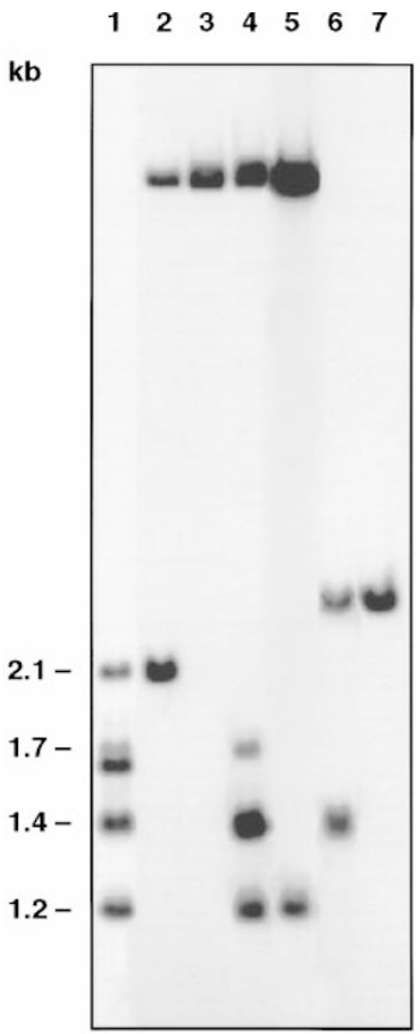

Figure 2 Southern blot of Hindlll-digested total human DNA and DNAs extracted from human $\times$ mouse and human $\times$ hamster cell lines, hybridised with exons 16,17 and 18 of the functional NFI gene. Lane 1: human lymphocytes; Lane 2 chromosome 17-only human $\times$ mouse cell line GM 10498B; Lane 3 mouse-only cell line Wehi-TG; Lane 4 chromosome 14-only human $\times$ mouse cell line Wegroth B3; Lane 5 chromosome 22-only human $\times$ mouse cell line PgMe25Nu; Lane 6 chromosome2-only human $\times$ hamster cell line GM 10826B; Lane 7 hamster-only cell line A3. 
For the NF1 pseudogenes on chromosomes 2 and 14, Southern blot analysis was performed using chromosome2only and chromosome14-only cell lines. Like for chromosome22, human-specific hybridising fragments were only detected for exons10b, 12a-19a, and 27b (data not shown). Except for exons 16, 17 and 18, all hybridising HindIII fragments observed with chromosomes 2 and 14 were of the same length as those with chromosome22. Exons 16, 17, and 18 hybridised on chromosome 22 with a fragment of $1.2 \mathrm{~kb}$, on chromosome 2 with a fragment of $1.4 \mathrm{~kb}$ and on chromosome 14 with fragments of $1.2,1.4$, and $1.7 \mathrm{~kb}$ (Figure2). The three signals in the chromosome14 lane were quantified. Assuming that the HindIII fragment of $1.7 \mathrm{~kb}$ represents one copy, we calculated that the fragments of 1.2 and $1.4 \mathrm{~kb}$ represent two and five copies, respectively. This suggests that eight copies of an NF1 pseudogene, which may differ from each other, are present on this chromosome 14.

To obtain clones with sequences from the NF1-related locus on chromosome2, we screened a chromosome 2 cosmid library (LL02NC02) with PCR products of exon 15, 17, and 19a. Only one positive cosmid was found, clone $180 \mathrm{H} 4$. All exons represented in the NF1 pseudogene on chromosome2 as well as the fusion of intron 19a to intron 27a were present in this cosmid and their sequences were determined (GenBank Accession Nos AF232292AF232302).

Since a chromosome14-specific library was not available, 15 clones containing NF1 homologous loci were obtained by screening a human female PAC library (RPCI6). Upon Southern blot analysis with exons 16, 17, and 18, seven of these clones showed HindIII fragments with a length of 1.2, 1.4 , or $1.7 \mathrm{~kb}$. FISH analysis revealed that these clones all hybridise to the pericentromeric regions of chromosome arms $22 q$ and $14 q$ and to $2 q 21$. In addition, the transition of intron 19a to intron 27a could be PCR-amplified from these seven PACs. Sequence analysis showed that three clones were derived from chromosome2. Two other clones carried sequences identical to those presented by Régnier et $\mathrm{al}^{11}$ as фNF1-14A (GenBank Accession Nos AF232248-AF232258) and $\phi N F 1-14 B$ (GenBank Accession Nos AF232259AF232269). A third clone, which we named $\phi N F 1-14 C$, showed a HindlII fragment for exons 16, 17, and 18 of the same length $(1.4 \mathrm{~kb})$ as the chromosome2 NF1 pseudogene (Figure2), but sequence analysis revealed that this clone originated from chromosome14 rather than from chromosome2 (GenBank Accession Nos AF232270-AF232280). The fourth clone, $\phi N F 1-14 D$ (GenBank Accession Nos AF232281-AF232291), yielded the $1.7 \mathrm{~kb}$ HindlI fragment for exons 16, 17 and 18, which is present on chromosome14, but absent on chromosomes 2 and 22 (Figure2).

Compared with the exons in the functional NF1 gene, several base substitutions, insertions and deletions occur in the exons represented in the pseudogenes on chromosomes2, 14, and 22. Neverthel ess, they share a high degree of identity, as shown in Figure3. Phylogenetic analysis was performed by maximum parsimony using exonic sequences of the functional NF1 gene and the NF1 pseudogenes, with the functional gene as outgroup. Analysis by the neighbourjoining method resulted in the same evolutionary tree as was found by maximum parsimony and the nodes were supported under the bootstrap test. Although bootstrap analysis of the most parsimonious tree did not yield significant values, the overall results suggest that the NF1 pseudogenes can be divided into two groups; one that is formed by the pseudogene on chromosome 2 and another by the pseudogenes on chromosomes 14 and 22.

To investigate whether the homologies between the various NF1 pseudogene regions extend beyond the NF1 gene duplicated segments, we determined the nucleotide sequence of flanking regions. For this purpose, primer pairs were

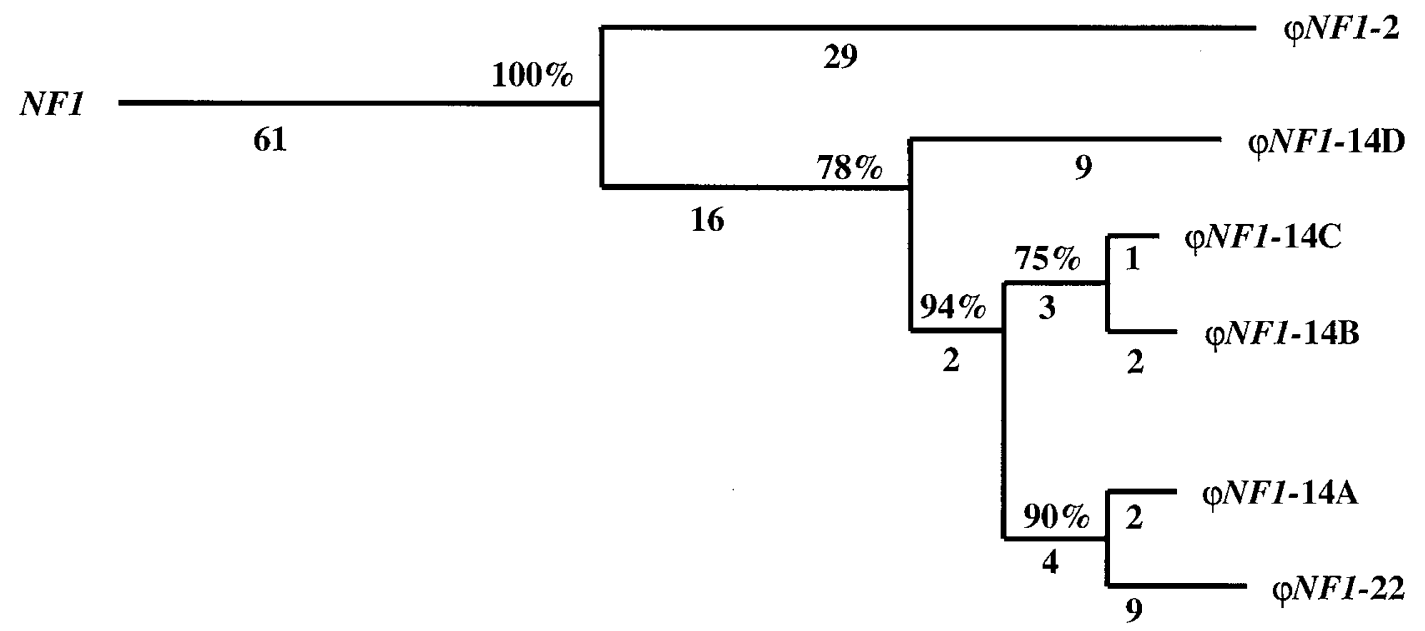

Figure 3 Phylogenetic tree of the functional NF1 gene and its pseudogenes on chromosomes2, 14, and 22. Branch lengths are indicated by numbers of nucleotide changes and are given below each branch. Bootstrap values are indicated above each branch as percentages of 1000 iterations. 
developed on the basis of the complete sequence of the NF1 pseudogene region on chromosome 22 and used to PCRamplify the corresponding segments in the clones containing the chromosome2- and chromosome14-NF1 pseudogenes. A BLAST search revealed that marker D22S111 (in 22q11.2) is located at about 1300 bp 5' of the NF1-homologous segment on chromosome22 (Figure1). The primers for D22S111 and those for region $\mathrm{A}$, at about $1800 \mathrm{bp} 3^{\prime}$ of the last segment with homology to the functional NF1 gene(Figure1), yielded PCR products in all clones. Each PCR product was sequenced and alignments of these sequences show at least 95\% homology (Figure4).

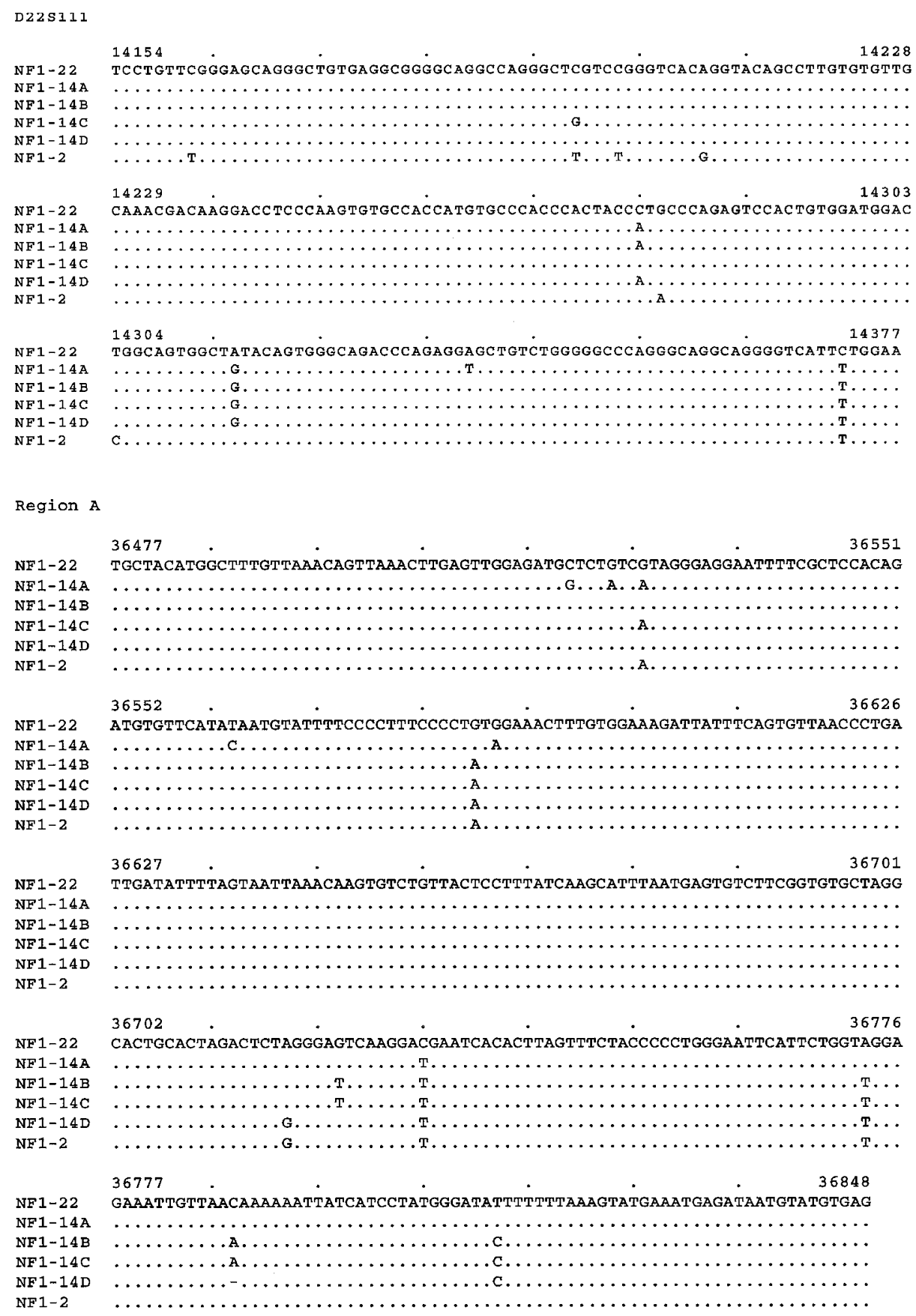

Figure 4 Alignments of sequences flanking the NF1 pseudogenes on chromosomes2, 14, and 22. Primers for marker D22S111 and for region A in, respectively, the 5' and 3' flanking regions of the NF1 pseudogene on chromosome22 (see Figure 1) were used to amplify by PCR the corresponding segments in the NF1 pseudogene regions on clones derived from chromosomes 2 and 14 . The resulting sequences were compared to the sequence of marker D22S111 and of region A on chromosome 22. 


\section{Discussion}

We have determined the complete sequence of the NF1 pseudogene on chromosome22. As is shown in Figure1, only the middle part of the functional NF1 gene is represented and complex rearrangements accompanied its insertion. Exons 19b-27a were deleted, resulting in the fusion of intron 19a to intron 27a. This deletion includes exons21$27 a$, which code for the functionally important GAP-related domain of the NF1 protein. Compared with the functional NF1 gene, the exon-intron structure of its representation in the chromosome 22 NF1 pseudogene is well conserved. The overall similarity between the sequenced regions of the functional gene and the pseudogene is $95 \%$, with no difference in mutation rate between exons and introns.

We have identified two new NF1 pseudogenes on chromosome14 ( $\phi N F 1-14 C$ and $\phi N F 1-14 D$ ) in addition to the two already known copies on that chromosome ( $\phi N F 1-14 \mathrm{~A}$ and $\phi N F 1-14 B) .4,7,11$ The phylogenetic tree in Figure 3 demonstrates that the new pseudogenes are highly related to the other pseudogenes on chromosomes2, 14, and 22. Moreover, they all contain the same exons and the transition of intron 19a to intron 27a, the latter marking the deletion of the GAP-related domain encoding exons. As such, they differ from other NF1 pseudogenes, especially those on chromosome 15, in which representation of GAP-related domain encoding exons has been demonstrated. ${ }^{3,5,10,11}$

Based on their phylogen etic tree, Régnier et $\mathrm{al}^{11}$ suggested a common ancestral sequence for the NF1 pseudogenes on chromosomes 2, 14, 15, and 22. Duplication and transposition of this ancestral sequence would have resulted in the NF1 pseudogenes that are now present on chromosomes 2 and 15. The results presented here indicate that this duplication process gave rise to NF1 pseudogene copies that differed al ready in the presence (chromosome2) or absence (chromosome15) of the deletion that includes the GAP-related domain encoding exons. Duplication and transposition of the NF1 pseudogene copy on chromosome 2 then may have resulted in the pseudogenes on chromosomes 14 and 22 . Further duplication and transposition might then most likely have generated the multiple copies of NF1 pseudogenes currently present on chromosome 14 .

We demonstrated more than $95 \%$ sequence conservation in regions flanking the 5'- and 3'- ends of the NF1 pseudogenes on chromosomes 2,14 , and 22 (Figure4). This high homology strongly suggests that during evolution of the ancestral NF1 pseudogene on chromosome2, the sequences flanking this locus were also included in the subsequent duplication and transposition processes. The sequenced segments display no homology at all with the corresponding regions in the functional NF1 gene on chromosome 17. This absence of homology may explain why our clones, contain- ing the NF1 pseudogenes on chromosomes 2, 14, and 22, did not reveal any in situ hybridisation with the functional NF1 gene on chromosome arm 17q, despite having 95\% sequence homology in the represented part, or with other NF1-related loci. FISH experiments with neighbouring clones are in progress to determine whether or not these cross-hybridise with the NF1 pseudogenes on chromosomes 2 and 14. These studies will enable estimation of the length of the duplicated and transposed segment.

\section{Acknowledgements}

This work was supported in part by NIH-NHGRI grant HG00313 to BAR. LJS and LD are supported by the Wellcome Trust. GenBank Accession Nos AC002471, AC003064, AC005374, AF232248AF232302.

\section{References}

$1 \mathrm{Li} \mathrm{Y,} \mathrm{O'Connell} \mathrm{P,} \mathrm{Huntsman} \mathrm{Breidenbach} \mathrm{H}$ et al: Genomic organization of the neurofibromatosis1 gene (NF1). Genomics 1995; 25: 9-18.

2 Xu G, O'Connell P, Viskochil D et al: The neurofibromatosistype 1 gene encodes a protein related to GAP. Cell 1990a; 62: 599-608.

3 Legius E, Marchuk DA, Hall BK et al: NF1-related locus on chromosome15. Genomics 1992; 13: 1316-1318.

4 Marchuk DA, Tavakkol R, Wallace MR et al: A yeast artificial chromosome contig encompassing the typel neurofibromatosis gene. Genomics 1992; 13: 672-680.

5 Gasparini P, Grifa A, Origone P, Coviello D, Antonacci R, Rocchi $M$ : Detection of a neurofibromatosis type1 (NF1) homologous sequence by PCR: implications for the diagnosis and screening of genetic diseases. Mol Cell Probes 1993; 7: 415-418.

6 Suzuki H, Ozawa N, Taga C, Kano T, Hattori M, Sakaki Y: Genomic analysis of a NF1-related pseudogene on human chromosome21. Gene 1994; 147: 277-280.

7 Purandare SM, Huntsman Breidenbach $\mathrm{H}, \mathrm{Li}$ Y et al: Identification of neurofibromatosis 1 (NF1) homologous loci by direct sequencing, fluorescence in situ hybridization, and PCR amplification of somatic cell hybrids. Genomics 1995; 30: 476-485.

8 Cummings LM, Trent JM, Marchuk DA: Identification and mapping of type1 neurofibromatosis (NF1) homologous loci. Cytogenet Cell Genet 1996; 73: 334-340.

9 Hulsebos TJM, Bijleveld EH, Riegman PHJ, Smink LJ, Dunham I: Identification and characterization of NF1-related loci on human chromosomes 22, 14 and 2. Hum Genet 1996; 98: 7-11.

10 Kehrer-Sawatzki H, Schwickhardt GT, Rocchi M, Krone W: A third neurofibromatosis type 1 (NF1) pseudogene at chromosome15q11.2. Hum Genet 1997; 100: 595-600.

11 Régnier V, M eddeb M, Lecointre G et al: Emergence and scattering of multiple neurofibromatosis (NF1)-related sequences during hominoid evolution suggest a process of pericentromeric interchromosomal transposition. Hum Mol Genet 1997; 6: 9-16.

12 Ijdo JW, Baldini A, Ward DC, Reeders ST, Wells RA: Origin of human chromosome2: an ancestral telomere-telomere fusion. Proc Natl Acad Sci USA 1991; 88: 9051-9055.

13 Hulsebos TJM, Gilbert DJ, Delattre O et al: Assignment of the $\beta B 1$ crystallin gene (CRYBB1) to human chromosome 22 and mouse chromosome5. Genomics 1995; 29: 712-718.

14 Roe BA, Crabtree JS, Khan AS: DNA Isolation and Sequencing: Essential Techniques Series. John Wiley: New York, 1996.

15 Weiss RB, Dunn DM, Aoyagi A et al: Genbank AC004526, 1998. 